\title{
Prognostic implications of qualitative assessment of left ventricular function compared to simple routine quantitative echocardiography
}

\author{
Paul B Silcocks, James F Munro, Richard P Steeds, Kevin S Channer
}

\begin{abstract}
Objective-To compare the prognostic value of qualitative estimates of left ventricular function with that of routine simple quantitative indices used in echocardiography.
\end{abstract}

Design-Retrospective follow up study.

Setting-University hospital.

Patients-The records of 2964 patients who had undergone echocardiography and who could be traced on the family health services register were examined; 919 cases were included in the study, and a further 458 were used to validate the statistical models for prognostic assessment. There were 928 exclusions on the basis of referral for or diagnosis of alternative conditions, and 659 because of incomplete collection of the qualitative and quantitative data used in the study.

Main outcome measure-Survival over the study period.

Results-A qualitative "eyeball" estimate of left ventricular function was of prognostic significance (relative risk of poor $v$ good, $2 \cdot 248 ; P<<0.001$; 95\% confidence interval 1.620 to $3 \cdot 119$ ). None of the quantitative echocardiographic indices was of independent prognostic significance when all variables were tested simultaneously in the regression model.

Conclusions-A qualitative echocardiographic estimate of left ventricular dysfunction is of prognostic value, supporting the view of many cardiologists who use their overall impression of left ventricular function at echocardiography as the basis for treatment decisions.

(Heart 1997;78:237-242)

Department of Public Health Medicine, University of Sheffield, Sheffield, UK

P B Silcocks

Medical Care Research

Unit, University of Sheffield, Sheffield,

UK

J F Munro

Department of

Cardiology, Royal

Hallamshire Hospital,

Sheffield, UK

R P Steeds

K S Channer

Correspondence to:

Dr Channer, Department of

Cardiology, Royal

Hallamshire Hospital,

Sheffield S10 2JF, UK.

Accepted for publication

2 June 1997

Cardiac failure is a common problem with a poor prognosis, with average five year survival of only $20 \% .^{1}$ Long term survival following myocardial infarction is directly related to left ventricular function, ${ }^{2}$ but may be improved with angiotensin converting enzyme inhibitors. ${ }^{3}$ Diagnosis of left ventricular dysfunction by clinical examination alone is inaccurate, ${ }^{45}$ while radionuclide angiography provides accurate prognostic information ${ }^{6}$ but at a cost to the patient of exposure to radioactivity and to the investigator of greater expense. It also has the disadvantage that the necessary equipment may not be available in a district hospital. Echocardiography provides a low risk procedure which is widely available at relatively low cost.

Various echocardiographic indices have been shown to have prognostic significance in cardiac failure and following myocardial infarction. These include the more simple estimates of left ventricular function derived from $M$ mode measurements, such as fractional shortening, ${ }^{78}$ but they have the disadvantage that they may underestimate damage where a chamber has been patchily affected. Cross sectional echocardiography has a similar prognostic value to radionuclide ventriculography for evaluating patients following myocardial infarction. ${ }^{9}$ However, detailed investigation of global left ventricular dysfunction takes time and algorithms may still overestimate ejection fraction when the ventricles are damaged segmentally. Many cardiologists appear to be influenced in their review of echocardiograms by an overall visual impression of ventricular function as opposed to a rigid interpretation based on quantitative measurements. Simple qualitative estimates of cardiac function have been shown to have a sensitivity of $82 \%$ and specificity of $86 \%$ when compared with quantitative cross sectional echocardiographic algorithms (using mainly the modified Simpson rule). ${ }^{5}$ There has been no previous study of the prognostic value of qualitative impressions of overall left ventricular function.

Our study was undertaken with three objectives: first, to determine whether qualitative echocardiographic estimates could provide reliable prognostic information; second, to examine the individual prognostic significance of various commonly used quantitative echocardiographic indices in typical patients referred for assessment of left ventricular dysfunction; and third, to establish whether the data collected routinely in the course of providing a district echocardiographic service were sufficiently detailed and complete to allow prognostic studies to be undertaken.

\section{Methods}

The echocardiographic service at the Royal Hallamshire Hospital is provided by trained cardiac technicians using a Toshiba SS130A cross sectional colour Doppler echocardiographic scanner. Scans are recorded onto videotape. Routinely collected measurements and final echocardiogram reports are recorded in a dedicated database.

From the database a cohort of patients was 
defined as those who had undergone at least one echocardiogram during the first five years of this service (1990 to 1994), and whose survival could be determined by reference to the patient register of the local family health services authority. This register records the date, but not the cause, of death. Patients not known to be dead were assumed to be alive at the censoring date of 31 January 1995.

The cohort was further restricted to those patients whose echocardiogram had been reported by a single consultant cardiologist (KSC), to improve consistency. Patients undergoing echocardiography for reasons other than assessment of left ventricular function, or those in whom the quality of the echocardiogram was recorded as "poor" at the time of the examination, were excluded from this cohort. Finally, the cohort was divided randomly into a "training set" (two thirds of subjects), which was used to develop the prognostic model, and a "validation set" (one third of subjects) which was used to assess it.

Seven quantitative echocardiographic indices were selected for analysis on the basis that these measurements would be taken in usual practice. These were left atrial diameter, aortic peak instantaneous gradient, left ventricular ejection fraction, left ventricular dimensions (posterior wall thickness, septal wall thickness), left ventricular hypertrophy, and the pressure gradient across the tricuspid valve derived from the peak velocity of the tricuspid regurgitant jet, when present.

For the echocardiographic assessment, left parasternal long axis, left parasternal short axis, and four and five chamber apical views were routinely obtained. Depending upon circumstances, high chest or epigastric windows were used.

Left atrial diameter was recorded from the $M$ mode image obtained in the left parasternal view through the aortic leaflets. ${ }^{10}{ }^{11}$ Aortic peak instantaneous gradient was measured from the continuous wave Doppler trace obtained from the aortic outflow tract and measured in the apical five chamber view. ${ }^{12}$ Left ventricular dimensions were measured from $M$ mode recordings taken at the level of the papillary muscles in the left parasternal view. Left ventricular ejection fraction was calculated by the Teicholz method from these measurements. (See appendix for details of this method.)

In addition to the quantitative measures, a qualitative "eyeball" assessment of each patient's left ventricular function was made by the reporting consultant, based on all views taken during echocardiographic examination. Left ventricular function was graded on a four point scale from normal to severe impairment,

Table 1 Qualitative assessment of left ventricular function

\begin{tabular}{|c|c|}
\hline Category & Definition \\
\hline $\begin{array}{l}\text { Normal } \\
\text { Mild dysfunction } \\
\text { Moderate dysfunction } \\
\text { Severe dysfunction }\end{array}$ & $\begin{array}{l}\text { All areas of myocardium contract normally } \\
\text { Segmental hypokinesia affecting less than } 25 \% \text { of the left ventricle } \\
\text { Segmental hypokinesia affecting } 25 \text { to } 50 \% \text { of the left ventricle } \\
\text { Global left ventricular hypokinesia, or segmental hypokinesia affecting } \\
\text { more than } 50 \% \text { of the left ventricle }\end{array}$ \\
\hline
\end{tabular}

on the basis of the worst function observed (table 1). Each patient's age and sex were also recorded. As is usual practice, the reporting consultant was aware of the reason given for requesting the test, but did not have access to the patient casenotes.

\section{STATISTICAL ANALYSIS}

Cox regression modelling was used to assess the prognostic value of the quantitative indices and the qualitative assessment. In order to determine whether variables should be treated as continuous or categorical in the model, univariate analyses were performed for each quantitative variable divided into its quintile groups (quartiles for the tricuspid regurgitant jet) to allow assessment of non-linear association. If this was non-significant, the variable was treated as a continuous linear measurement, with the exception of left ventricular hypertrophy which was graded as present or absent. The "normal limits" of these measurements were therefore not relevant.

The resulting model was checked in two ways: (1) the predictive value of the model was assessed using Harrell's c statistic, an extension of Kendall's Tau $_{\mathrm{a}}$ rank correlation to censored data; (2) the validity of using a proportional hazards model in the training set was checked by plotting a $\log (-\log )$ plot of survival in the three prognostic groups.

Finally, the model was used to divide the patient cohort into three prognostic groups, for which survival curves were plotted.

\section{Results}

An initial cohort of 2964 patients met the inclusion criteria for the study. Subsequently, we excluded 780 patients who had undergone echocardiography for a reason other than assessment of left ventricular dysfunction, 148 patients in whom the quality of the echocardiogram was recorded as "poor" at the time of the examination, and a further 659 patients for whom one or more variables was missing.

The characteristics of the resulting cohort of 1377 patients are shown in table 2 . In all, 215 patients had died by the censoring date. For those who survived, the maximum period of follow up was 1818 days ( 4.98 years) and the minimum was 307 days ( 0.84 years).

The cohort was then divided randomly into a "training set" of 919 cases and a "validation set" of 458 cases.

\section{COX REGRESSION}

In the Cox regression year of echo was fitted first as a factor, since it was assumed that the case mix would have changed over the period of service development. The effect of year was highly significant $\left(\chi^{2}\right.$ on $4 \mathrm{df}=30 \cdot 04$, $\mathrm{P}<<0.001)$. All further analyses were therefore adjusted for this variable.

The qualitative "eyeball" assessment made by the reporting consultant, though expressed as one of four categories, in fact showed a clear distinction between normal/mild impairment (relative risk (RR) 1.00 and 1.035 ) on the one hand and moderate/severe impairment ( $R R$ 
Table 2 Characteristics of the patient cohort

\begin{tabular}{|c|c|c|}
\hline & Male & Female \\
\hline $\begin{array}{l}\text { Number } \\
\text { Demographic characteristics } \\
\text { Age groups (years) } \\
15 \text { to } 24 \\
25 \text { to } 34 \\
35 \text { to } 44 \\
45 \text { to } 54 \\
55 \text { to } 64 \\
65 \text { to } 74 \\
75 \text { to } 84 \\
85 \text { and over } \\
\text { Inpatients, } n(\%) \\
\text { Deaths during follow up, } \mathrm{n}(\%)\end{array}$ & $\begin{array}{l}22 \\
24 \\
31 \\
88 \\
164 \\
209 \\
149 \\
27 \\
384(54) \\
115(16)\end{array}$ & $\begin{array}{l}14 \\
39 \\
38 \\
50 \\
113 \\
193 \\
177 \\
39 \\
348(53) \\
100(15)\end{array}$ \\
\hline $\begin{array}{l}\text { Echocardiographic characteristics } \\
\text { Left atrial diameter (mm), } \\
\text { median (interquartile range) }\end{array}$ & 41 (35 to 46$)$ & 38 (32 to 44$)$ \\
\hline $\begin{array}{l}\text { Posterior wall thickness (mm), } \\
\text { median (interquartile range) }\end{array}$ & $11(9$ to $12 \cdot 3)$ & 10 (8 to 12$)$ \\
\hline $\begin{array}{l}\text { Septal wall thickness (mm), } \\
\text { median (interquartile range) }\end{array}$ & $13(11$ to 15$)$ & 12 (10 to 14$)$ \\
\hline $\begin{array}{l}\text { Left ventricular ejection fraction (\%), } \\
\text { median (interquartile range) }\end{array}$ & 63 (50 to 72$)$ & 68 (57 to 76$)$ \\
\hline $\begin{array}{l}\text { Aortic peak instantaneous gradient ( } \mathrm{mm} \mathrm{Hg} \text { ), } \\
\text { median (interquartile range) }\end{array}$ & 4 (3 to 5$)$ & 5 (3 to 6$)$ \\
\hline $\begin{array}{l}\text { Tricuspid regurgitant jet, } \\
\text { median }\end{array}$ & 0 & 0 \\
\hline $\begin{array}{l}\text { Left ventricular hypertrophy (\%) } \\
\text { Moderate or severe LV dysfunction on "eyeball" } \\
\text { assessment (\%) }\end{array}$ & $\begin{array}{l}18 \\
25\end{array}$ & $\begin{array}{l}14 \\
13\end{array}$ \\
\hline
\end{tabular}

Table 3 Results of Cox regression of patient and echocardiographic variables

\begin{tabular}{lllll}
\hline Variable & $\begin{array}{l}\text { Beta } \\
\text { (Loge }(\text { relative risk)) }\end{array}$ & $\begin{array}{l}\text { Standard } \\
\text { error }\end{array}$ & P value & $\begin{array}{l}\text { Relative risk } \\
\text { (95\% CI) }\end{array}$ \\
\hline Age & 0.059 & 0.008 & $<0.0001$ & $1.06(1.04$ to 1.08) \\
"Eyeball" assessment & 0.810 & 0.167 & $<0.0001$ & $2.25(1.62$ to 3.12) \\
Patient source & 0.900 & 0.207 & $<0.0001$ & $2.46(1.64$ to 3.69) \\
\hline
\end{tabular}

"Exact" treatment of ties (adjusted for year).

$\star$ Per additional year of age.

$\mathrm{CI}$, confidence interval.

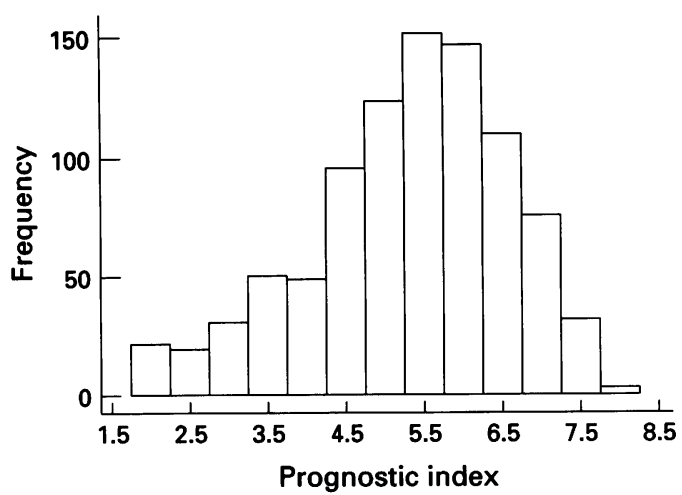

Figure 1 Distribution of values of the prognostic index in the training set: all years combined.

MODEL CHECKING

The predictive value of the Cox regression model was assessed using Harrell's c, a nonparametric method which is essentially the proportion of pairwise comparisons between all subjects in which the relative ranking of survival times is concordant with the ranking predicted from the prognostic index. ${ }^{13}$ It can also be thought of as a generalisation of the area under the receiver operating characteristic (ROC) curve, ${ }^{14}$ so that a value of 0.5 indicates random concordance and a value of 1 indicates perfect concordance of rankings. For the training set $\mathrm{c}$ was 0.795 while for the validation set it was $0 \cdot 790$, indicating moderate discriminating power. The extent to which the random allocation of subjects to the validation set might have affected the corresponding value of $c$ was found by a resampling procedure. ${ }^{15}$ This yielded an almost perfect normal distribution with a standard deviation of 0.0595 , giving $95 \%$ confidence limits of 0.73 to 0.85 for $\mathrm{c}$ in the validation set.

Age, referral source (inpatient or outpatient) and eyeball assessment of left ventricular function were all statistically significant independent predictors of survival (adjusted for year), although the effect of age was weak. The effect of referral source showed that inpatients had a clearly worse prognosis than outpatients, as might be expected.

Only two quantitative variables were associated with outcome on univariate analysis: left ventricular ejection fraction $(P<<0.001)$ and left atrial diameter $(P=0.0219)$. However these variables were no longer statistically significant if source, age, and eyeball assessment were already in the model, leaving no quantitative measure as a significant prognostic factor.

The final model is shown in table 3. As an additional check, even when all variables were included in the model, with left ventricular ejection fraction and left atrial diameterwhich had both been significant on univariate analysis-treated as categories in order to maximise any non-linear effect, there was little change in $\beta$ coefficients for source, age, and eyeball assessment. These all remained highly significant predictors of survival, while the $\chi^{2}$ for all the additional variables together was non-significant $\left(\chi^{2}=7 \cdot 38, \mathrm{df}=14, \quad \mathrm{P}=\right.$ $0.92)$. Sex was not significant in any of the analyses.

\section{PROGNOSTIC GROUPING}

The regression model defines a prognostic index for each individual patient, calculated from the $\beta$ coefficients of the Cox model referral source and the eyeball assessment of their echocardiogram. Thus:

$$
\begin{gathered}
\mathrm{PI}=0.059 \cdot \operatorname{age}(\text { years })+0.81 \cdot \text { eyeball }+ \\
0.9 \cdot \text { source }
\end{gathered}
$$

where "eyeball" normal/mild left ventricular dysfunction $=0$, moderate/severe dysfunction $=1$, and outpatient $=0$, inpatient $=1$.

Figure 1 shows the distribution of the prognostic index in our cohort. Higher values of the prognostic index imply a worse prognosis.

As the distribution of values of the prognostic index was unimodal, that is, with no obvious natural separation, three groups were arbitrarily defined using tertiles of the prognostic index in the training set, which were 4.91 and 5.97. Survival for the three groups so defined (all years combined) is shown in fig 2, using the same cutoff values for both training and validation sets.

Almost the whole of the "good" prognosis while about $90 \%$ of the "moderate" and $60 \%$ applied to the corresponding values of age, group was alive at three years (1096 days), 
Figure 2 Survival of good, moderate and poor groups, defined by tertiles of prognostic index.
A Training set

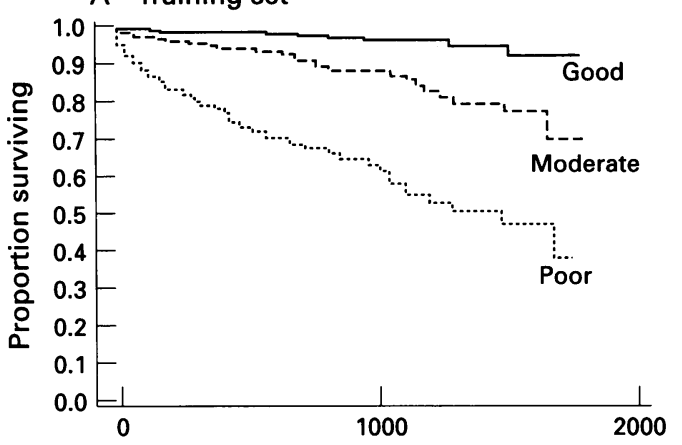

B Validation set

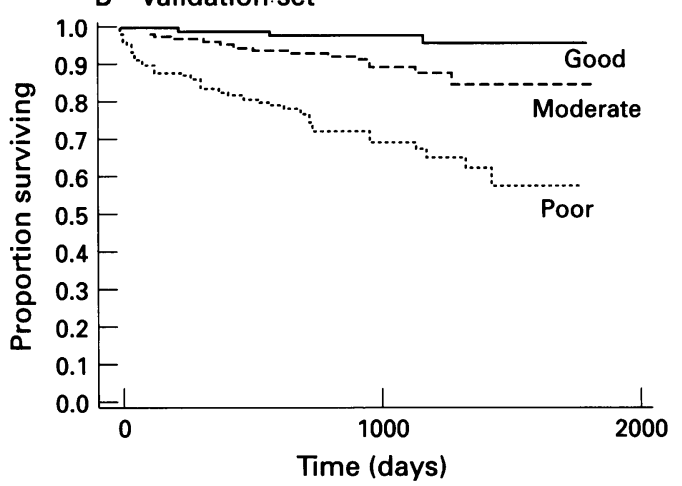

of the "poor" groups survived this period. In its present form, the index is evidently best at the distinction between "poor" and "not poor" but, as previously indicated, the discriminating power is only moderate. A rough check on the proportional hazards assumption of the regression model was made with a $\log (-$ $\log$ ) plot of survival in the prognostic groups of the training set. For all years except the fifth this condition was reasonably fulfilled.

\section{Discussion}

In a university hospital setting, none of the seven echocardiographic indices investigated here, all of which are recorded routinely in the assessment of patients referred with left ventricular dysfunction, was found to have prognostic significance. Left ventricular ejection fraction calculated by the Teicholz method was weakly associated with survival but this study suggests that a better assessment may be made by a simple visual rating of overall ventricular motion.

By convention, echocardiographic measurements from $M$ mode recordings are taken through a single slice of the ventricle at the tips of the papillary muscles. The measurements used in this study included septal and posterior wall thickness, left ventricular hypertrophy and the Teicholz calculation of ejection fraction. Each of these is susceptible to inaccuracies because of variation in transducer positioning on the chest wall, beat to beat variability in dimensions, and the effects of respiration. Furthermore, there may be difficulty correctly identifying the septal and posterior endocardial boundaries on which measurements are based.

The findings of this study accord with those of Baker et $a l,{ }^{16}$ who found no prognostic sig- nificance in the measurement of septal and posterior wall thickness in symptomatic cardiac failure. Although the Teicholz method may be the optimum $M$ mode method for estimation of ejection fraction, ${ }^{17}$ we suggest a number of reasons why it does not emerge as an important independent prognostic factor. The Teicholz method makes four fundamental assumptions: that the ventricle approximates an ellipse; that it contracts symmetrically along the major axis with little shortening in the major dimension; that the internal dimension measures the maximum circumference; and that the major axis is twice the minor axis dimension. However, both in normal systole and in the presence of left ventricular dysfunction, the left ventricular shape is actually more variable and less ellipsoid than assumed. Left ventricular wall thickening is unequal in different regions of the normal ventricle and this variation will be more marked in the diseased ventricle. More importantly, echocardiographic measurements of stroke volume and ejection fraction may overestimate myocardial function because of patchy damage. Difficulties also arise where there is left bundle branch block or right ventricular dilatation, which produce incoordinated septal contraction or paradoxical septal motion. Indices of function based upon single measurements may then be incorrect because recordings are taken in areas of actively contracting ventricle. The Teicholz measurement has not been studied before for its prognostic significance in unselected patients referred for assessment of left ventricular function, and we believe it fails because of the frequency of regional motion defects in the typical hospital population.

Left atrial diameter and aortic peak instantaneous gradient are quantitative variables which have previously been found to have prognostic significance after myocardial infarction and in dilated cardiomyopathy. ${ }^{1011}$ Our larger study has failed to confirm this, but there are differences in the study methodologies. Our study population comprised unselected patients referred for assessment of left ventricular function and no other selection criteria based on underlying pathology were applied, so it is likely to have been more heterogeneous than those of previous studies. Left atrial diameter is a single measurement from $M$ mode recordings and is susceptible to the errors of measurement we have described above. The aortic peak instantaneous gradient, although related to left ventricular output, is also affected by other factors such as the effective valve area. In particular, age is known to have an important influence on Doppler flow velocities. ${ }^{18}$ Interpretation of Doppler measurement of the aortic peak instantaneous gradient is problematic in some clinical settings. Previous studies have shown that both an increase in preload and a reduction in afterload may result in increased Doppler aortic peak velocity. ${ }^{19}{ }^{20}$ For example, in a patient with ischaemic left ventricular failure an expected decrease in aortic peak instantaneous gradient may be masked by a 
decrease in afterload, resulting from nitrate treatment, which would lead to an overestimation of function.

Aortic peak instantaneous gradient and peak velocity of the tricuspid jet share the limitation that they are late indicators of left ventricular dysfunction. Peak aortic gradient is only impaired in those with advanced left ventricular failure. Tricuspid regurgitant jet velocity would be expected to alter when there is right ventricular dilatation secondary to the development of pulmonary hypertension or in association with septal disease. This change occurs later in the natural history of failure and is likely to be a marker only of long standing or severe disease. The velocity of the tricuspid regurgitant jet is affected by changes in atrioventricular flow which occur during respiration and with changes in heart rate. We have shown that these two Doppler indices are poor prognostic discriminators in an unselected population referred for assessment of function. Further development of three dimensional and contrast echocardiography may allow the application of other quantitative indices of left ventricular function which could be used with greater accuracy.

In addition, there are two important limitations to this study which should be borne in mind when comparing our results with those of previous reports. First, potential prognostic variables related to the patient and their clinical management were not included in the model. While age-which was included-was an independent predictor of mortality, we did not take into account risk factor profile, aetiology, intercurrent illness, or alteration of treatment following echocardiography. Furthermore, in the absence of data on cause of death, all cause rather than cause specific mortality is the end point of our analysis. Second, we have not yet examined the question of variability in patient handling during the investigation. All echocardiograms were reported by a single consultant but the measurements were made by different technicians. It is possible that interobserver variability was sufficiently great to negate the prognostic significance of the quantitative indices examined. Previous studies have found considerable inter-test variability of $M$ mode variables such as end diastolic diameter and septal and posterior wall thickness. ${ }^{21-23}$ In comparison with measurements from magnetic resonance imaging, echocardiographic estimates of left ventricular mass vary by an average of $11 \%$ (SD 6.4\%). ${ }^{24}$ However, we have no reason to suppose that the measurements made in our unit are inherently more variable than those of routine practice in a district general hospital department, suggesting that these results are relevant to everyday clinical practice. We are currently investigating inter-test variability of both quantitative and qualitative assessments, which will be the subject of a future report.

Our report is of the type which Simon and Altman term a "phase 2" study-that is, it was exploratory and hypothesis generating, yielding results which need confirmation. ${ }^{25}$ For example, while the values for $\mathrm{c}$ indicate only moderate discrimination, further studies will be needed to prove whether such a value is in fact typical in practice. Our results certainly show that routinely collected diagnostic data can be used for prognostic studies. However, survival analysis involves much more than the estimation of Cox regression coefficients. Even variables that have a strong, statistically significant association with survival may not have great discriminating power in terms of predicting outcome. Models need to be checked to ensure that basic assumptions are met, or at least not seriously violated, and allowance should be made for "overoptimism" in the sense that a model is bound to fit best to the data that generated it.

\section{CONCLUSIONS}

In this study, qualitative assessment of left ventricular function has been shown to have individual prognostic significance in a standard hospital setting. Since qualitative assessment depends upon a global impression of function gained from all echocardiographic views obtained, it is plausible that such an assessment would be better at predicting survival in an unselected group of patients, including those with patchily damaged ventricles, than quantitative measures which reflect single elements of ventricular structure or function.

The obvious disadvantage of the eyeball assessment is that it is likely to be very dependent on the reporter's experience and, if used more widely, would require each echocardiographer's ability to assess left ventricular function to be subject to some form of quality assurance. However, our study has also shown that it is possible to link routinely collected survival data to the results of clinical investigations and it might therefore be possible to audit a reporter's capability in a similar way. A qualitative assessment of wall motion index has previously been shown to have prognostic significance in cardiac failure, ${ }^{26}$ and recently treatment based upon wall motion index has been shown to improve outcome. ${ }^{27}$ It would seem that qualitative measures of left ventricular function by skilled echocardiographers may be more useful in routine practice than the quantitative methods specified by more complicated intervention trials, and it should be remembered that much of clinical examination, radiology, and histopathology is also founded on subjective assessment. However, the value of our index must finally depend on whether others find it of practical use.

\section{Appendix}

The ejection fraction is calculated from the measured left ventricular dimensions using the Teicholz formula, as follows:

End diastolic volume $(\mathrm{EDV})=$

$$
\frac{7 \times \text { LVIDD }^{3}}{2 \cdot 4+\text { LVIDD }^{-}}
$$

End systolic volume $(\mathrm{ESV})=$

$$
7 \times \text { LVIDS }^{3}
$$


Where LVIDD = end diastolic ventricular dimension and LVIDS = end systolic dimension. Then stroke volume $=\mathrm{EDV}-\mathrm{ESV}$, and ejection fraction $(\%)=$ stroke volume/end diastolic volume $\times 100$.

1 Kannel WB, Belanger AJ. Epidemiology of heart failure. Am Heart $\mathcal{F}$ 1991;121:951-7.

2 The Multicentre Post Infarct Research Group. Risk stratification and survival after myocardial infarction. $N$ Engl $\mathcal{f}$ Med 1983;309:331-6.

3 Pfeffer M, Braunwald E, Moye L, Basta L, Brown E, Cuddy $T$. Effect of captopril on mortality and morbidity in patents with left ventricular dyfunction after myocardial infarction. The SAVE Trial. N Engl $f$ Med 1992;327: 669-77.

4 Remes J, Miettinen H, Reunanen A, Pyorala K. Validity of clinical diagnosis of heart failure in primary care. Eur clinical diagnosis of heart

5 Choy A-M, Darber D, Lang C, Pringle T, McNeill G, Kennedy $N$, et al. Detection of left ventricular dysfuncion after acute myocardial infarction:comparison of clinical, echocardiographic and neurohormonal methods. $\mathrm{Br}$ Heart f 1994;72:16-22.

6 The SOLVD Investigators. Effect of enalapril on survival in patients with reduced left ventricular ejection fractions and congestive heart failure. $N$ Engl f Med 1991;325: 293-302.

7 Galderisi M, Lauer M, Levy D. Echocardiographic determinants of clinical outcome in subjects with coronary artery disease (the Framingham Heart Study). $A m$ f Cardiol 1992;70:1971-6.

8 Creager M, Faxon D, Halperin J, Melidossian C, McCabe $C$, Schick $\mathrm{E}$, et al. Determinants of clinical response and survival in patients with congestive heart failure treated with captopril. Am Heart $₹$ 1982;104:1147-54.

9 Van Reed R, Quinones M, Poliner L, Nelson J, Waggoner A, Kanon D. Comparison of 2D echocardiography with gated radionuclide ventriculography in the evaluation of 243-52.

10 Eriksson S, Caidahl K, Hamsten A, de Faire U, Rehnqvist $\mathrm{N}$, Lindvall $\mathrm{K}$. Long term prognostic significance of $M-$ mode echocardiography in young men after myocardial infarction. Br Heart f 1995;74:124-30.

11 Sachero A, Cassazza F, Recalcati F, de Maria R, Preti L, Mattioli $R$, et al. Clinical and prognostic significance of echocardiographic parameters in dilated cardiomyopathy: a prospective study on 225 patients. The Italian Multicentre Study of Cardiomyopathies. Giomale Ital Cardiol 1992;22:1077-90.

12 Delemarre B, Visser C, Bot H, de Koning H, Dunning A. Predictive value of pulsed Doppler echocardiography in acute myocardial infarction. $f \mathrm{Am}$ Soc Echocardiogr 1989; 2:102-9.
13 Harrell F, Lee K, Califf R, Pryor D, Rosati R. Regression modelling strategies for improved prognostic prediction.

14 Hanley J, McNeill B. The meaning and use of the area under a receiver operating characteristic curve. Radiology 1982;143:29-36.

15 Efron B, Tibshirani R. Bootstrap methods for standard errors, confidence intervals and other measures of statistical accuracy. Stat Sci 1986;1:54-77.

16 Baker B, Leddy C, Galie N, Casebolt P, Franciosa J. Predictive value of $\mathrm{M}$-mode echocardiography in patient with congestive heart failure. Am Heart $\mathcal{f}$ 1986;111: 697-702.

17 Kronik G, Slany J, Mosslacher H. Comparative value of eight $M$-mode echocardiographic formulas for determining left ventricular stroke volume. Circulation 1979;60: ing left vent

18 Gardin JM, Burn CS, Childs WJ, Henry WL. Evaluation of blood flow velocity in the ascending aorta and main pulmonary artery of normal subjects by Doppler echocardiomonary artery of normal subjects by Dopp

19 Harrison MR, Clifton GD, Berk MR, DeMaria AN. Effect of blood pressure and afterload on Doppler echocardioof blood pressure and afterload on Doppler echocardiographic measurements of systolic finc
jects. Am $₹$ Cardiol 1989;64:905-8.

20 Bedotto JB, Eichhorn EJ, Grayburn PA. Effects of left ventricular preload and afterload on ascending aortic blood velocity and acceleration in coronary artery disease. $A m \mathcal{F}$ Cardiol 1989;64:856-9.

21 Stefadouros M, Canedo M. Reproducibility of echocardiographic estimates of left ventricular dimensions. $\mathrm{Br} \mathrm{Heart}$ $\mathcal{F} 1977 ; 39: 390-8$.

22 Stollberger C, Hollander I, Dimitrov L, Slany J. Influence of measurement inaccuracies on determination of left ventricular mass by $M$-mode echocardiography. Hear 1996;75:312-3.

23 Szlachic J, Massie B, Greenberg B, Thomas D, Cheitlin M Bristow J. Intertest variability of echocardiography and chest radiography measurements: implications for decision-making in patients with aortic regurgitation. $\mathcal{f} \mathrm{Am}$ Coll Cardiol 1986;7:1310-7.

24 Germain P, Roul G, Kastler B, Mossard J, Bareiss P, Sacrez A. Inter-study variability in left ventricular mass measurement: comparison between $M$-mode echocardiomeasurement: comparison between M-mode ech.

25 Simon R, Altman DG. Statistical aspects of prognostic factor studies in oncology. Br f Cancer 1994;69:979-85.

26 Kuhn Madsen B, Egeblad H, Hojberg S, Melchior T, Videbaek R, Sorum C, et al. Prognostic value of echocardiography compared to other clinical findings. Multivariate analysis based on long-term survival in 456 patients. Cardiology 1995;86:157-62.

27 Køber L, Torp-Pedersen C, Carlsen JE, Bagger H, Eliasen $\mathrm{P}$, Lyngborg $\mathrm{K}$, et al. A clinical trial of the angiotensin converting enzyme inhibitor trandolapril in patients with left ventricular dysfunction after myocardial infarction. $N$ Engl F Med 1995;333:1670-6. 